\title{
Temporal and spatial effects in luminance discrimination'
}

\author{
SIDNEY STECHER ${ }^{2}$ \\ BRANDEIS UNIVERSITY
}

\begin{abstract}
Luminance difference thresholds $(\Delta I)$ were obtained by a successive and a simultaneous method of presenting the stimuli. Spatial and temporal separation between the fields was the independent variable while other variables as stimulus size, luminance, retinal area of stimulation and duration were kept constant. $\Delta I$ was less for simultaneously presented stimuli than for successively presented stimuli. This was related to spatial and temporal interaction effects such that the greater the spatial interaction between simultaneously presented fields, the greater the discriminability while the greater the temporal interaction between successively presented fields, the less the sensitivity to luminance differences. It was suggested that the basis of the luminance discrimination may be different under conditions of temporal interaction than under conditions of spatial interaction between the fields.
\end{abstract}

Several investigators have distinguished between simultaneous and successive stimulus presentation methods of obtaining the luminance difference threshold $(\Delta \mathrm{I})$ (Durup \& Fessard, 1938; Bartlett, 1942; LeGrand, 1957) and the nature of the bases of the discrimination underlying these methods. As a major difference between these two methods is the extent of spatial and temporal interaction between the fields, it is possible that the luminance discrimination data will reflect these differences. Under suitably arranged conditions, it may be possible to assess the influence of these spatial and temporal interaction effects on luminance discrimination.

In the simultaneous method, two temporally synchronous and spatially adjacent stimuli, differing only in luminance, are presented to the observer for comparison. This stimulus configuration has also commonly been used to investigate simultaneous contrast effects in vision (Diamond, 1953; Heinemann, 1955) and Stecher (1968) has recently suggested a possible relationship between luminance discrimination measured by a simultaneous comparison method and simultaneous contrast.

The successive method differs from the simultaneous in that two temporally asynchronous and equal size stimuli are presented to the same retinal location (within the limits of fixation accuracy) for comparison. This method appears to have first been introduced by McDougall (1904) for making apparent brightness matches. It has, however, not been subjected to much systematic analysis, although recently Nachmias and Steinman (1965) have made use of the technique and Stecher (1967) has reported a study in which luminance discrimination as a function of the dark interval and luminance was investigated.

Previous investigations of luminance discrimination have used mainly the "increment" method in which the task of the $S$ is to detect the presence of a small increment field of luminance, the latter being superimposed on a larger background field which is usually, although not always (Crawford, 1947), turned on before the increment is presented (see Graham, 1966, for review). The increment method can, therefore, be considered as a successive comparison task. The use of this particular method, however, makes it difficult to analyze the effect of successive versus simultaneous methods of stimulus presentations and temporal versus spatial interaction effects on discriminability since the luminance gradient existing between the successively presented increment and the background field leads to marginal contrast effects (Mach Bands) (Ratliff, 1965).

LeGrand $(1957$, p. 268) has pointed out that a number of investigators have found that the luminance difference thresholds obtained by the two presentation methods (simultaneous versus successive) are not very different if determined under similar conditions. Durup and Fessard (1938) however, present the thesis that the simultaneous presentation of stimuli is influenced by induction effects and that the successive method gives a better estimate of differential sensitivity.

Bartlett (1942) has also drawn a distinction between two ideal types of discrimination based on simultaneous and successive stimulus presentation modes. He points out on logical grounds that "The mechanism for the comparison of the brightnesses of two areas is possibly quite unlike that for comparing the brightness of an area at one time with its brightness an instant later" (p. 382). His study, however, is not addressed to the assessment of this problem. More recently, Blackwell (1963) has pointed to the possibility of different types of discrimination in stating that "a different discriminatory process is involved in the stimulus-increment and the stimulus comparison experiments" ( $p$. 141).

Further evidence that the mechanisms underlying simultaneous and successive comparison of stimuli may be different is found in a study reported by Cornsweet and Pinsker (1965). They found that simultaneously presented stimuli yielded a Weber function which was horizontal for five $\log$ units above the absolute threshold while the increment (successive) presentation mode yielded a more traditional Weber function which increased at low luminances. Cornsweet and Pinsker, however, used stimulus fields which were spatially separated by $1 \mathrm{deg} 10 \mathrm{~min}$. There is evidence to indicate that spatial and temporal interaction effects in the visual system influence the perception of spatially and temporally separated stimuli (Barlow, 1958; Beitel, 1934). It is probable that spatial or temporal separations existing between visual fields presented either simultaneously or successively will influence the capacity of the observer to make luminance discrimination judgments and that this influence will be reflected in the shape of the Weber function $(\Delta I / I)$ obtained.

The elimination of the temporal factor can easily be achieved by presenting a bipartite field for simultaneous comparison and this arrangement has been used to study various parametric influences on discriminability (Bartlett, 1942). The "gated" stimulus presentation technique of successive comparison to a large extent eliminates luminance gradients and hence the occurrence of marginal contrast effects (within the limits of fixation accuracy).

In the present investigation, stimulus area and duration were kept constant for foveally presented fields and variations in the Weber function were assessed by studying: (1) the influence of temporal separation on two equal size $(1 \mathrm{deg}) 10 \mathrm{msec}$ visual fields presented to the same foveal area in succession, and, (2) the influence of spatial separation between the halves of a bipartite (1 deg) $10 \mathrm{msec}$ simultaneously presented foveal field (see Smith, 1936).

The results indicate that the Weber functions obtained under simultaneous versus successive stimulus presentation conditions are highly dependent on the separations existing between the fields and that the underlying basis of the discriminations may be different under those conditions producing temporal interaction from under those conditions leading to the spatial interaction between visual fields.

\section{APPARATUS}

A schematic diagram of the apparatus used in both experiments is shown in Fig. 1.

The system is arranged for monocular Maxwellian viewing by the right eye of the $S$ and is of conventional design. The light source $(S)$ is a projection lamp with a horizontal ribbon filament (Westinghouse EDW TIO SR6A). The source was run at a constant current of 17.5 A supplied by an Electro Model H Filtered dc power supply. Detailed description of the optical paths has been described elsewhere (Stecher, 1967, 1968). 


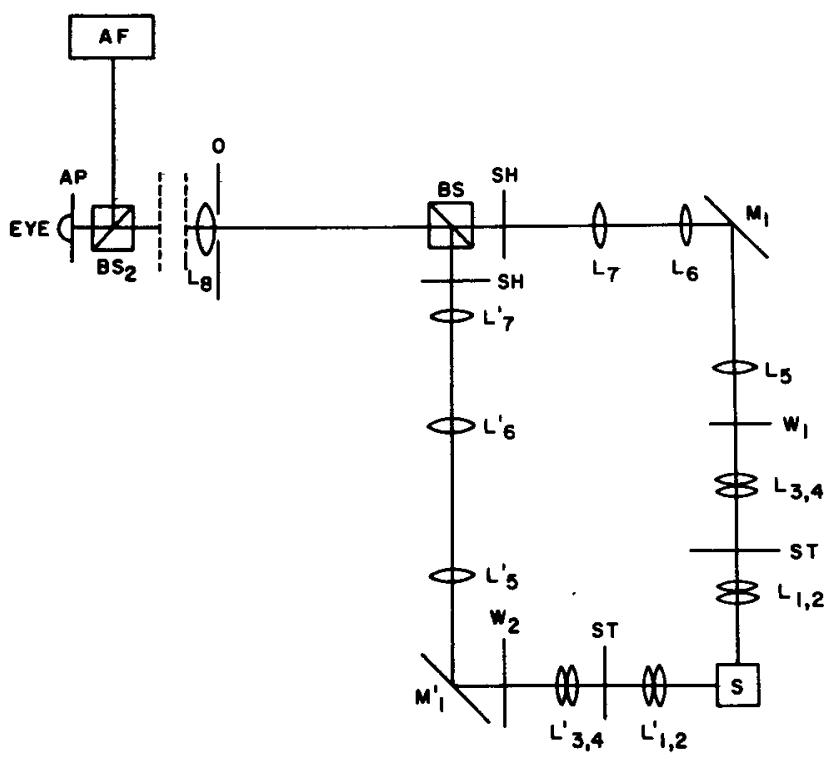

Fig. 1. Diagram of optical system used in the experiments.

\section{PROCEDURE}

\section{Experiment 1-Temporal Separation}

In this experiment two stimuli that differed only in luminance were presented in succession to the same area of the retina and the S's basic task was to report which one appeared brighter. The principal variables studied were the length of the dark interval separating the flashes and the luminance at which the measurements were made.

$\mathrm{S}$ dark adapted for $15 \mathrm{~min}$ by wearing an eye patch over his right eye and then looked at the $.008 \mathrm{~mL}$ adapting field for another $5 \mathrm{~min}$. After removing the eye patch $E$ determined a $\Delta I$ (difference threshold) on each of two Ss by the method of limits (Guilford, 1936; Woodworth \& Schlosberg, 1954) with an ascending and descending series at each of four luminance levels one logarithmic unit apart from each other and at each of nine interstimulus temporal intervals (ISI). ISIs were measured from the cessation of pulse one to the onset of pulse two. Data are presented for one $S$ and three interstimulus intervals but the results are typical for those found for other ISIs and the other S.

For each presentation of the $10 \mathrm{msec}$ pair of pulses, the second of the pair was set at a standard value (one of the four luminances used) on Channel 2 in the optical system and the S's task was to indicate whether the first of the pair appeared brighter, equal to, or dimmer than the second. In order to eliminate any effects due to knowledge of the series, E occasionally repeated a presentation and accepted the second of the two responses if a difference existed. Fixation was foveal and $S$ presented the stimuli to himself after waiting $30 \mathrm{sec}$ between exposures.

Between 20 and 28 such threshold determinations at each luminance and interstimulus interval were undertaken.

\section{Experiment 2-Spatial Separation}

In Experiment 2 two adjustable rectangular fields differing only in luminance and shown simultaneously were presented for 10 msec to S's right eye. The basic task of the $S$ was to indicate which side appeared brighter.

In this study a $\Delta I$ was determined using the conventional method of limits for five levels of luminance in log unit steps and for eight spatial separations between the fields. The $S$ sat, as in the temporal study, dark adapted and with head held in position by a dental biting board. The exposure of the stimuli was done by $S$ after a ready signal was given by $\mathrm{E}$. $\mathrm{S}$ waited $30 \mathrm{sec}$ between presentations.

The lighted halves were separated in space by various opaque angular subtenses. On half the trials the standard (I) was presented on the left, while the variable $(I+\Delta I)$ field was to the right. On the other half of the trials the situation was reversed. S was instructed to report whether the variable field appeared brighter, equal to, or dimmer than the standard which was placed along side of it. The orders of ascending or descending, repetition of exposure, standard on left or right, and the angular subtense of the separation between 0.5 deg halves were all randomized.

\section{Calibration Procedures}

Relative density of the wedges and filters were measured with a Macbeth illuminometer and a Spectra Pritchard Photometer (Model No. 1970-PR). Density values were also checked with phototube (RCA-929) corrected by a Kodak Wratten filter (No. 106). Absolute luminance calibration of the optical channels was made by photometrically matching each of the fields to an external standard whose luminance was known. Pulse durations and ISI were periodically monitored by using a CRO and a phototube. Durations and asynchronies of repeated rectangular pulses were found to be reliable to within $\pm 1 \mathrm{msec}$. All viewing was done through a $2.75 \mathrm{~mm}$ artificial pupil.

\section{RESULTS AND DISCUSSION}

Figure 2 demonstrates the effect of spatial and temporal separation between two fields on the logarithm of the Weber fraction.

$\log \Delta I / I$ is plotted as a function of $\log I$ for three selected values of temporal separation intervening between two successively presented superimposed $1 \mathrm{deg}, 10 \mathrm{msec}$ visual fields and for three values of spatial separation intervening between two simultaneously presented $0.5 \mathrm{deg}, 10 \mathrm{msec}$ fields. Values for the other spatial and temporal functions are presented elsewhere (Stecher, 1967, 1968).

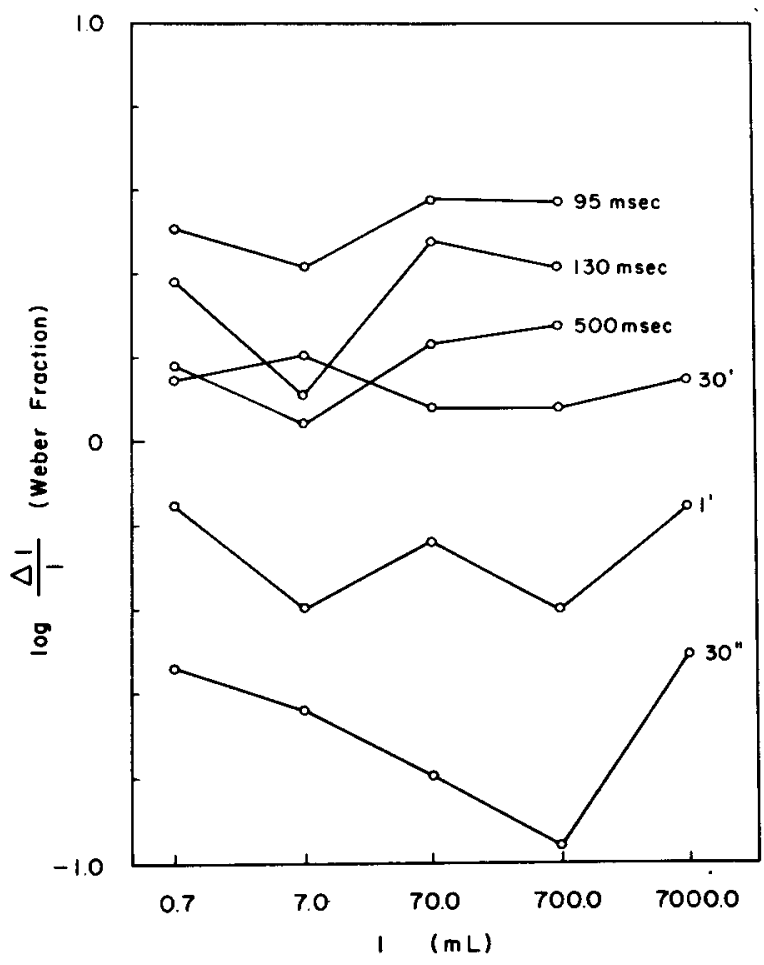

Fig. 2. Logarithm of the Weber fraction $(\Delta / / I)$ as a function of logarithm of the base luminance (I) in $\mathrm{mL}$. The parameter of the top three curves is the duration of the interval between the cessation of the first $10 \mathrm{msec}$ flash and the onset of the second 10 msec flash (ISI) (each field subtending $1 \mathrm{deg}$ ) for the successive presentation method. The parameter of the bottom three curves is the spatial separation between a simultaneously presented $10 \mathrm{msec}$ bipartite field (each illuminated half being $0.5 \mathrm{deg}$ ). 
The three lowest functions represent the $\log$ Weber fraction obtained when the two visual fields were simultaneously presented. The parameter of these three curves is the spatial separation which existed between the fields to be discriminated from one another. These separations were considered to be small (30 sec), intermediate (1 min), and large (30 min).

The three uppermost functions have as their parameter a long (500 $\mathrm{msec})$, intermediate (130 $\mathrm{msec}$ ), and short $(95 \mathrm{msec})$ temporal interval existing between the two successively presented 1 deg fields between which a luminance discrimination was made.

It is obvious from this plot that stimuli presented simultaneously are more easily discriminated from one another than are successively presented stimuli. This argues strongly against LeGrand's (1957) assertion that discriminability is not very different for the two methods if other conditions are kept comparable. This is especially so since the durations $(10 \mathrm{msec})$ and the total stimulation areas ( $1 \mathrm{deg}$ ) were kept constant in the present series of studies.

If one considers that the visual angle of the variable field in the simultaneous presentation method was $0.5 \mathrm{deg}$, and that the variable field was 1 deg for the successive method the absolute magnitude of the differences becomes even more pronounced for the two methods. That is, the larger area would lead one to expect a lower value of $\Delta \mathbf{I}$ for successive than for simultaneously presented fields. Further, one would even expect that the values of $\Delta \mathrm{I}$ for the $500 \mathrm{msec}$ curve would be somewhat lower than the $\mathbf{3 0}$ $\sec$ function. That this is not the case is demonstrated rather unambiguously in Fig. 2.

These functions also argue against the point made by Durup and Fessard (1938) that because of induction effects in simultaneous stimulus presentation the successive method gives a better estimate of differential sensitivity. It is more likely that the underlying basis of the discrimination is different in each of these situations and quite probable that simultaneous contrast effects aid discriminability when stimuli are simultaneously presented (Stecher, 1968).

There appears to be a progressive shift in the spatially separated functions such that with a narrow separation between the fields, sensitivity is at its best for all luminances of the standard. With a progressively greater separation between the fields relative differential sensitivity decreases. It is also appasent that as the separation between the simultaneously presented fields increases, the shape of the Weber function changes from marked curvature to an increasingly horizontal trend. The function obtained with a $30 \mathrm{sec}$ separation between the fields exhibits a progressive decrease in the Weber fraction to a luminance of $700 \mathrm{~mL}$ and a subsequent rise, while the function obtained using the widest separation (30 $\mathrm{min}$ ) is relatively horizontal. The extent of the variation in the Weber fraction can be seen to be almost $1 / 2$ of a logarithmic unit for the $30 \mathrm{sec}$ function while it was just slightly over $1 / 10$ of a $\log$ unit under conditions of wide separation $(30 \mathrm{~min})$. It should be remembered that the daily variability of the judgments as well as the variability in the photometric calibration of the Maxwellian system ranged up to but was no more than about $3 / 10$ of a logarithmic unit. If these errors of measurement are taken into consideration, the gradual trend toward horizontality becomes apparent in the simultaneously presented spatially separated Weber functions.

It was previously stated that Cornsweet and Pinsker (1965) obtained a Weber function which was horizontal for five log units upward from the absolute threshold in their simultaneously presented stimulus condition. The spatial separation between their stimulus fields was $1 \mathrm{deg} 10 \mathrm{~min}$. This is quite concordant with the present results as the progressive tendency for the function to become horizontal with an increase in the separation between the simultaneously presented fields would lead to the prediction that Cornsweet and Pinsker's results would be horizontal.

The three topmost functions are those obtained by successively presenting the stimuli to be discriminated from one another. It was found that with a wide temporal interval $(500 \mathrm{msec})$ between the successive fields discrimination was better than with a short (95 msec) intervening "dark" period, although this difference was not as dramatic as that found for simultaneous viewing conditions.
The difference between the lowest value of $\log \Delta \mathrm{I} / \mathrm{I}$ for a long temporal separation $(500 \mathrm{msec})$ and the lowest value obtained for short temporal separation $(95 \mathrm{msec})$ under successive presentation conditions was somewhere on the order of $1 / 2$ of a logarithmic unit. Comparison of the difference between the lowest value of $\log$ $\Delta \mathrm{I} / \mathrm{I}$ for a narrow spatial separation $(30 \mathrm{sec})$ and the highest value obtained for a wide spatial separation $(30 \mathrm{~min})$ indicates a difference on the order of $1-2 / 10$ log units. The differences due to spatial interaction between simultaneousiy presented visual fields thus appear much mory extensive than those differences due to temporal interaction.

The shape of the Weber function was generally not found to change as a function of the interstimulus interval. The Weber functions all appeared to be horizontal, especially if one takes into account the errors of measurement previously mentioned.

A plausible reason for the lack of apparent replication of the results obtained by Cornsweet and Pinsker may be found in the range of luminances investigated in both studies. Cornsweet and Pinsker found an increase in the Weber fraction at the very lowest luminances investigated. These luminances were not investigated in the present study and the lack of agreement with these earlier authors may be more apparent than real.

Unlike the simultaneous comparison situation where the log Weber function was found to increase as the spatial separation between the fields increased, the $\log$ Weber function for successively compared stimuli increased as the temporal separation between the fields decreased. In other words, as the spatial interaction between the fields increased, differential luminance sensitivity also increased, but as the temporal interaction between the fields increased, differential luminance sensitivity decreased. Furthermore, both wide spatial ( $30 \mathrm{~min}$ ) and long temporal ( 500 msec) separations yielded Weber functions which were, within the limits of measurement error, indistinguishable (in shape and value). Thus, to the degree that the interaction between two fields is involved in supra-threshold luminance discrimination these data would imply that spatial and temporal interaction are opposite in the direction of their effects on discriminability. It would appear that the spatial interaction between the fields enhances the detectability of luminance differences while the temporal interaction between the fields hampers luminance discrimination and that the underlying basis of the discrimination is different under simultaneous as opposed to successive comparison conditions.

One possible simple explanation of the results of the simultaneously compared stimuli has been previously proposed (Stecher, 1968) and is related to the theory held by Heinemann (1961) and suggested earlier by Fechner. Heinemann proposes that the relevant variable governing luminance discrimination data is not luminance or brightness but the rate at which brightness changes under particular stimulation conditions. The data presented in this paper were not intended to test this theory but the thought is presented that perhaps the rate of change of brightness changes as a function of the temporal and spatial interaction between the fields to be discriminated from one another. There is evidence in studies of simultaneous contrast (Leibowitz, Mote, \& Thurlow, 1954) that as simultaneously presented fields are spatially separated from one another, the interaction between the test and inducing fields tends to decrease. As the experimental arrangement used in the simultaneous comparison experiment presented here was identical with the arrangement used for investigation of simultaneous contrast, one can conclude that relatively similar mechanisms must be operative under both situations. If a constant brightness difference were required in order for a difference to be detected between the fields, then, from what is known concerning simultaneous contrast, one would require a greater luminance difference between the fields to yield a constant apparent brightness difference when the fields were far apart than when they were close together. One must also, when considering simultaneously presented stimuli between which a luminance difference exists, consider the relative contribution of Mach bands to discrimination (Ratliff, 1965).

With successively presented stimuli the relative contribution of successive contrast and the role this plays in discrimination must be assessed. It is plausible to assume that the apparent brightness 
of both the first and second flash changes as a function of the interstimulus interval. It is possible that successive luminance discrimination data are related to the apparent brightness difference of the two flashes and that the rate of change of the apparent brightness difference between the flashes changes with changes in the interstimulus interval. This matter is currently under investigation as is the effect of the after-image due to the first flash on the discrimination of the luminance difference between two successive flashes.

\section{REFERENCES}

BARLOW, H. B. Temporal and spatial summation in human vision at different background intensities. J. Physiol., 1958, 141, 337-350.

BARTLETT, N. R. The discrimination of two simultaneously presented brightnesses. J. exp. Psychol, 1942, 31, 380.

BLACKWELL, H. R. Neural theories of simple visual discriminations. J. Opt. Soc. Amer., 1963, 53, 129-160.

CORNSWEET, T., \& PINSKER, H. M. Luminance discrimination of brief flashes under various conditions of adaptation. J. Physiol, 1965, 176, 294-310.

CRAWFORD, B. H. Visual adaptation in relation to brief conditioning stimuli. Proc. Roy. Soc. (London), 1947, B-134, 283-302.

DIAMOND, A. L. Foveal simultaneous brightness contrast as a function of inducing and test field luminances. J. exp. Psychol. 1953, 45, 304-314.

DURUP, G., \& FESSARD, A. Revue critique: Des seuils differentiels "successifs" et presentation d'un appareil a plage unique. Ann. Psychol, 1938, 39, 227.

GRAHAM, C. H. Vision and visual perception. New York: Wiley, 1965. Chap. 9.

GUILFORD, J. P. Psychometric methods. New York: McGraw-Hill, 1936.

HEINEMANN, E. G. The relation of apparent brightness to the threshold for differences in luminance. J. exp. Psychol, 1961, 61, 389-399.

HEINEMANN, E. G. Simultaneous brightness induction as a function of inducing and test field luminances. J. exp. Psychol, 1955, 50, 89-96.

LeGRAND, Y. Light, colour and vision. Translated by R. W. G. Hunt, J. W. T. Walsh, and F. R. W. Hunt. New York: Wiley, 1957.

LEIBOWITZ, H., MOTE, F. A., \& THURLOW, W. R. Simultaneous contrast as a function of separation between test and inducing fields. $J$. exp. Psychol, 1953, 46, 453-456.

MCDOUGALL, $w$. The variation of the intensity of visual sensation with the duration of the stimulus. Brit. J. Psychol, 1904, 1, $151-189$.

NACHMIAS, J., \& STEINMAN, R. Brightness and discriminability of light flashes. Vis. Res, 1965.

RATLIFF, F. Mach Bands: Quantitative studies on neural networks in the retina. San Francisco: Holden-Day, 1965.

SMITH, J. R. Spatial and binocular effects in human intensity discrimination. J. gen. Psychol, 1936, 14, 318-345.

STECHER, S. Luminance difference thresholds and simultaneous contrast. Amer. J. Psychol, March, 1968.

STECHER, S. Discrimination of luminance differences between temporally separated paired flashes. J. Opt. Soc. Amer., 1967, 57, 1271-1272.

WOODWORTH, R. S., \& SCHLOSBERG, H. Experimental psychology. New York: Holt, 1954.

\section{NOTES}

1. This research was done while the author was at Brooklyn College (CUNY) and was supported by an NSF grant to Eric G. Heinemann.

2. Address: Department of Psychology, Brandeis University, Waltham, Mass. 02154.

(Accepted for publication May 30, 1968.) 